\title{
A New Joint Antenna Selection Algorithm Based on Capacity
}

\author{
Li Yuan ${ }^{\text {a, }}{ }^{* 1}$, Liu Ping ${ }^{\text {a, }}{ }^{* 2}$, Li Jianlan ${ }^{\text {a }}$ \\ ${ }^{a}$ School of electronic information, Tianjin University, Tianjin, 300072, China
}

\begin{abstract}
Antenna Selection in MIMO systems can increase the system capacity, reduce the MIMO system complexity and cost of radio links effectively. In this paper, a new joint antenna selection algorithm was presented which can adaptively change the number of the selected transmitting and receiving antenna number according to the channel station. It can obtain the similar system capacity with the optimal joint antenna selection algorithm in any correlation coefficient, but the calculated amount is far less than the optimal joint algorithm.
\end{abstract}

Index Terms: MIMO System; Joint Antenna Selection Algorithm; Correlation Coefficient; Interrupted Capacity

(C) 2011 Published by MECS Publisher. Selection and/or peer review under responsibility of the Research Association of Modern Education and Computer Science

\section{Introduction}

MIMO is the product of the combination of antenna diversity and space-time processing techniques in areas of wireless mobile communications. It originates from the antenna diversity and smart antenna technology and owns both the advantages of them. MIMO technology uses multiple antennas in the transmitter and receiver end and it effectively develops the use of space dimension [1]. Thus, it can increase capacity of the system without increasing the transmitter power and bandwidth and can multiply increase the efficiency of spectrum utilization. But this performance is due to the multi-antenna configuration which calls for equipping appropriate RF link to each transmitting and receiving antenna in the corresponding end. The cost of hardware will increase significantly and the configuration and maintenance of the system will be difficult when there are too many base station users. Moreover, with the increasing of the number of antennas the complexity of encoding and decoding algorithms increases, which is very detrimental to the real-time transmission of information.

Antenna selection technique is an effective solution [2]. The performance of the system can be guaranteed and the channel capacity has little influence after antenna selection. Studies have shown that the use of a good antenna selection algorithm can increase the cost-effective of the system. As the study on the antenna selection continuing, literature [3-7] separately proposed some different algorithms. The optimal antenna selection algorithm described in literature [3] achieved optimal performance, but the calculated amount is too huge. Literature [4] is the antenna selection algorithm based on the maximum F-norm, which greatly reduces

\footnotetext{
* Corresponding author:

| E-mail address: ${ }^{* 1}$ liyuan@ @ tju.edu.cn; ${ }^{* 2}$ liuping050826@163.com
} 
computation. However, Compared with the optimal algorithm, the channel capacity is very poor on the condition of channel-related or a higher SNR (signal to noise ratio). Literature[5-6] are two sub-optimal antenna selection algorithm with regard to criteria for maximum Shannon capacity--incremental antenna selection algorithm and decreasing antenna selection algorithm. These two algorithms can get similar capacity with the optimal algorithm. But as the increasing of antenna number, the computation of the algorithm is also considerable. Literature [7] studied the antenna selection algorithm from the relevant point. The advantage of this algorithm is not needed to know the SNR of the receiver and the computation has been significantly reduced, but the channel capacity is very poor.

The above algorithms generally select the number of the fixed antennas and most make selection to only one of receiving or transmitting end of the antenna. The joint selection for transmit and receive antennas is sparse, which is very unfavorable to complex wireless communication environment.

In this paper, the antenna selection algorithm in the MIMO system has been analyzed and summarized from the perspective of maximizing channel capacity. Based on these existing algorithms, a new joint antenna selection algorithm is proposed and compared with the existing algorithms.

Simulation results show that the improved joint antenna selection algorithm can not only provide the similar channel capacity to the optimal joint algorithm ,but also change the number of the selected receiving antennas adaptively .In case of low correlation between antennas, the new algorithm uses more antennas to achieve higher channel capacity; on contrast, the new algorithm can reduce the number of antennas to meet the requirements of system capacity. In addition, the algorithm has low computational complexity to meet the requirements of the real-time transmission.

\section{System Model}

MIMO channel is assumed as quasi-static Rayleigh fading channel, $N_{R} \times N_{T}$ gives that the channel matrix elements are independent and identically distributed complex Gaussian variables and the mean and variance are 0 and 1 respectively. The transmit and receive correlation matrix can be definite as $R_{T X}$ and $R_{R X}$, then the related MIMO channel matrix can be expressed as

$$
H=R_{R X}{ }^{1 / 2} H_{W} R_{T X}^{1 / 2}
$$

Antenna selection algorithm selects actual $L_{R}$ receive antennas and $L_{T}$ transmit antennas from $N_{R}$ receive antennas and $N_{T}$ transmit antennas respectively, $0<L_{R} \leq N_{R}, \quad 0<L_{T} \leq N_{T}$. The channel matrix $L_{R} \times L_{T}$ is expressed as $H_{S}$, then the MIMO channel input-output relationship is

$$
y=\sqrt{\frac{E_{S}}{N_{T}}} H_{S} \cdot x+n
$$

y is $N_{R} \times 1$ - dimensional vector of received signal ,x is $N_{T} \times 1$-dimensional of transmit signals, $n_{\text {is }}$ ZMCSCG noise and the covariance matrix is $\varepsilon\left\{n n^{H}\right\}=N_{0} I_{N_{R}}, E_{S}$ is total average power that can be got from the transmitter in a symbol cycle. 
The new algorithm proposed in this paper is a joint antenna selection algorithm that transmits firstly and then receives. Supposing a MIMO system: transmitter has $N_{t}$ antennas and $L_{t}$ RF chains, receiver has $N_{r}$ and $L_{r}$ respectively. The information state of the channel is known to receiver but unknown to transmitter .The power of transmitting antenna is distributed evenly. Assuming that the symbols on each antenna element are not relevant and has normalized power. The channel has the characteristics of flat fading and it is the frequency non-selective fading channel. On one realized condition of the channel matrix $H$, we select $L_{t}$ columns from $N_{t}$ columns and $L_{r}$ lines from $N_{r}$ lines to realize the maximum capacity of the MIMO system.

\section{New Joint Antenna Selection Algorithm}

The new algorithm is a gradually decreasing algorithm. At the transmitting end, the antenna with the largest norm correlated coefficient is removed. It's selected by the antenna selection method based on norm and correlation. At the receiving end, the variable antenna selection based on capacity is used to select the number of antennas according to the different correlated coefficient. The standard of capacity selection is controlled by SNR. In general, the new algorithm is a objective joint antenna selection algorithm with variable number of antennas. The ideology of the antenna selection based on norm correlation is to select the antenna whose channel matrix $H$ has biggest norm correlated coefficient and then delete it. Continue doing this until the number of the columns of the channel matrix is $L_{t}$ [8]. The definition of the parameters between the column vector $\alpha_{i}, \alpha_{j}$ is

$$
\mu_{i, j}=1-\left(\frac{\alpha_{i}^{H} \alpha_{j}}{\left\|\alpha_{i}\right\|\left\|\alpha_{j}\right\|}\right)^{2}
$$

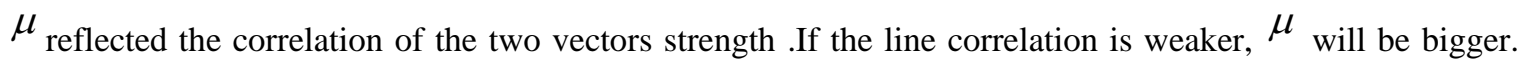
The transmitter takes the size of the relevant parameters $\chi_{n, m}$ as standard. You have to delete an antenna which makes the biggest $\chi_{n, m}$.

$$
\chi_{n, m}=\sum_{i=1}^{N_{t}}\left(\left\|\alpha_{(i)}\right\| \sum_{j=1}^{N_{t}} \mu_{i, j}\right)
$$

The capacity-based antenna selection algorithm is used at the receiving end. With the changing of the correlation of the antennas and SNR of the system, the selection criterion is the ratio of system capacity of the selected antenna and the maximum system capacity. That is $C_{S_{R}} / C_{\max }>\beta, 0<\beta<1$ [9].

The steps of new antenna selection algorithm is : 
1. Estimate the complete channel state matrix $H$;

$$
w_{r}=\left(1,2, \cdots, N_{r}\right), w_{t}=\left(1,2, \cdots, N_{t}\right)
$$

2. Estimate the column correlation and norm; estimate the receiving SNR and define $\beta$.

3. Set the initial value: $L_{r}=L_{t}=n, L_{R}=N$. $w_{r}=\left(1,2, \cdots, N_{r}\right)$ and $w_{t}=\left(1,2, \cdots, N_{t}\right)$ represents the selected receiving and transmitting antenna respectively; $C_{\max }=\log _{2} \operatorname{det}\left(I+\rho H H^{H} / N_{T}\right)$ indicates the largest capacity after transmitter selection; $H_{s}=H$ represents channel matrix of selected antenna;

4. Calculate each column norm $\|h(:, i)\|\left(1 \leq i \leq N_{t}\right)$ of $H$ and the correlation coefficient $\mu$ of every two of them; Calculate $\chi_{n, m}$, find out the three smallest; Judge $\beta$.

5. According to the new matrix $H w$ composed by three smallest arrays and $C_{\max }=\log _{2} \operatorname{det}\left(I+\rho H_{w} H_{w}{ }^{H} / N_{T}\right)$ While $C_{S_{R}} / C_{\max }>\beta$, the antenna which makes the smallest contribution to the channel capacity is deleted. Update $w_{r}=\left(1,2, \cdots, N_{r}\right), H_{s}=H ; L_{R}=L_{R-1}$;

$$
C_{S_{R}}=\log _{2} \operatorname{det}\left(I+\rho H_{s} H_{s}{ }^{H} / L_{R}\right) \text {; }
$$

End ;

\section{Performance Simulation and Analysis}

Suppose a MIMO system with six transmitting antennas and six receiving antennas in Rayleigh fading channels. Other conditions are the same as mentioned above. Because of the limited antenna spacing or signal extension angle, the receiving antennas may have a relatively high correlation, but the correlation between the transmitting antennas can be ignored. So the related MIMO channel can be expressed as

$$
H=R_{r}^{1 / 2}(f) H_{W}
$$

The 10,000 channel matrix samples generated randomly is $H_{W}$. Each element of the channel matrix $H_{W_{i, j}}\left(1 \leq i \leq N_{R}, \quad 1 \leq j \leq N_{T}\right)$ is a complex Gaussian random variable whose mean is 0 , and the variance is 1 . It takes SNR as variable to compare the distribution of the capacity derived from various algorithms when correlated coefficient in the receiving end changes.As there are two ways to represent capacity in the MIMO systems, that is traverse capacity and outage capacity[10], the paper take outage capacity to represent capacity and select the $10 \%$ outage capacity.

\subsection{Analysis of correlation coefficient}

We can see from Fig 1 to 4: First of all, the new algorithm and the optimal joint algorithm have similar capacity in the same channel state regardless of the correlated coefficients. With the correlated coefficient increasing, the capacities are almost similar. Secondly, the capacity derived from the new algorithm is bigger than that derived from receiving optimal algorithm and the joint receiving and transmitting random algorithm 
regardless of the correlated coefficient. In addition, the capacity of the new algorithm doesn't decrease much and the system outage capacity is the same as the optimal joint algorithm when the correlated coefficient is large. Therefore, the new algorithm is very suitable for the uncertain correlation coefficient in the receiving end. Since there is not huge of matrix operations at the transmitter and it uses regressive algorithm the calculated amount is far less than the optimal joint algorithm. So it's suitable for real-time transmitting.

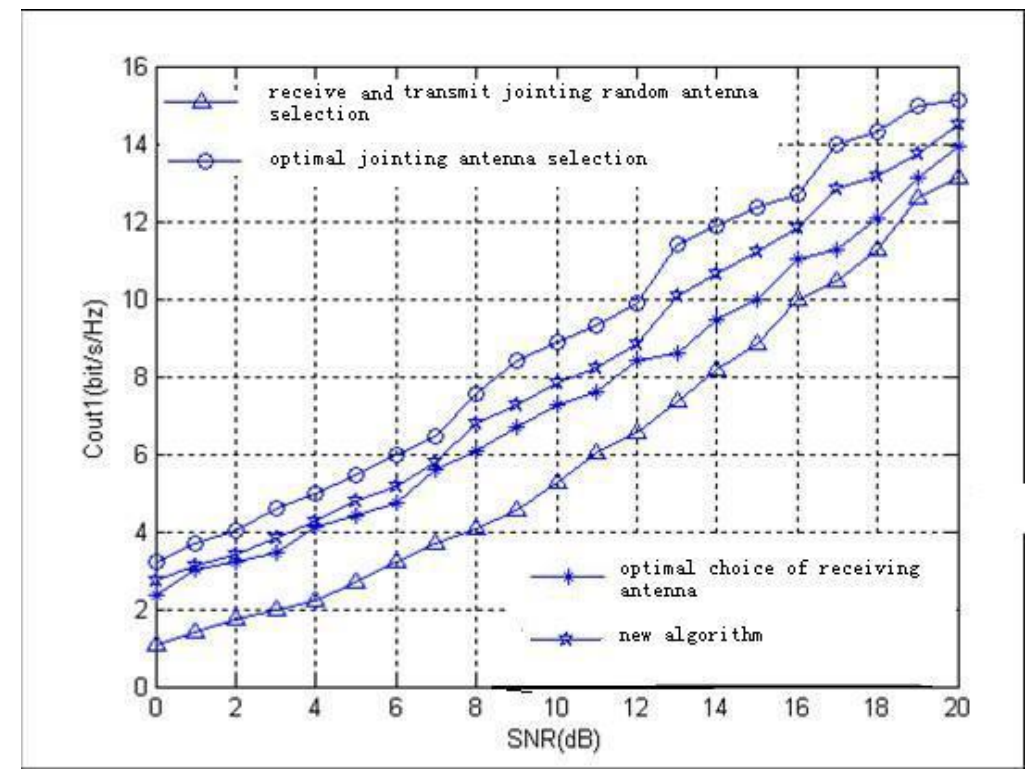

Fig. 1 . The $10 \%$ outage capacity when the correlation coefficient is 0 .

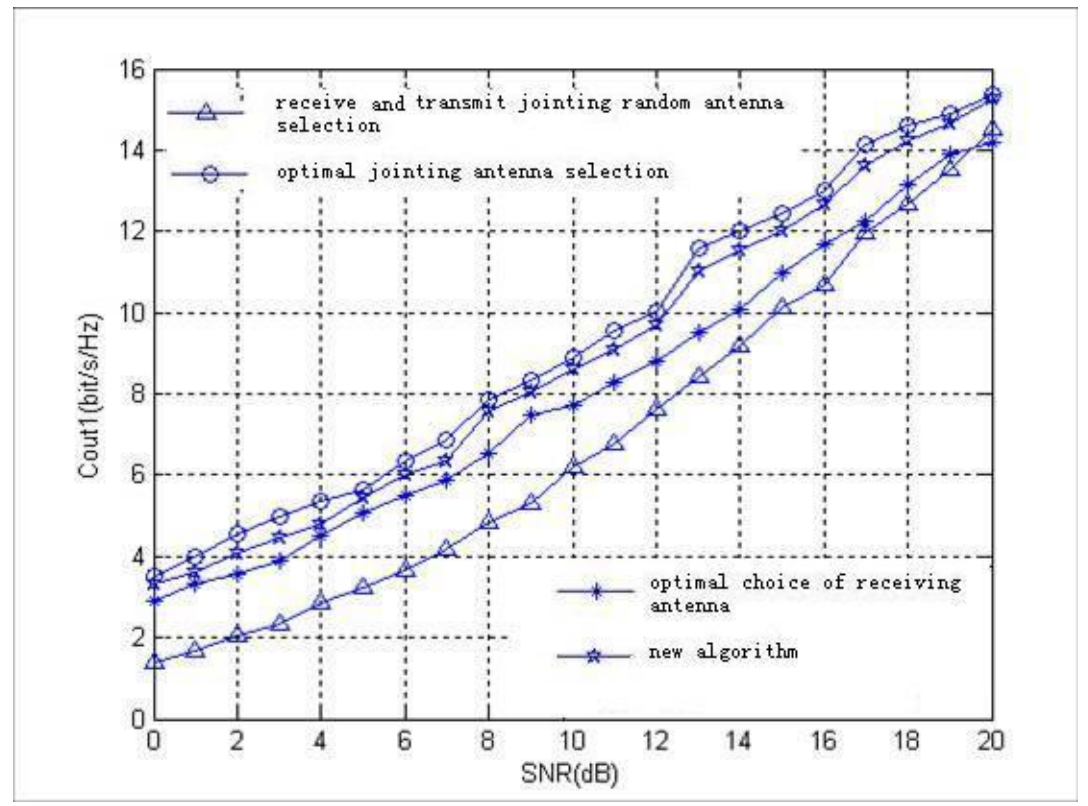

Fig. 2. The $10 \%$ outage capacity when the correlation coefficient is 0.2 


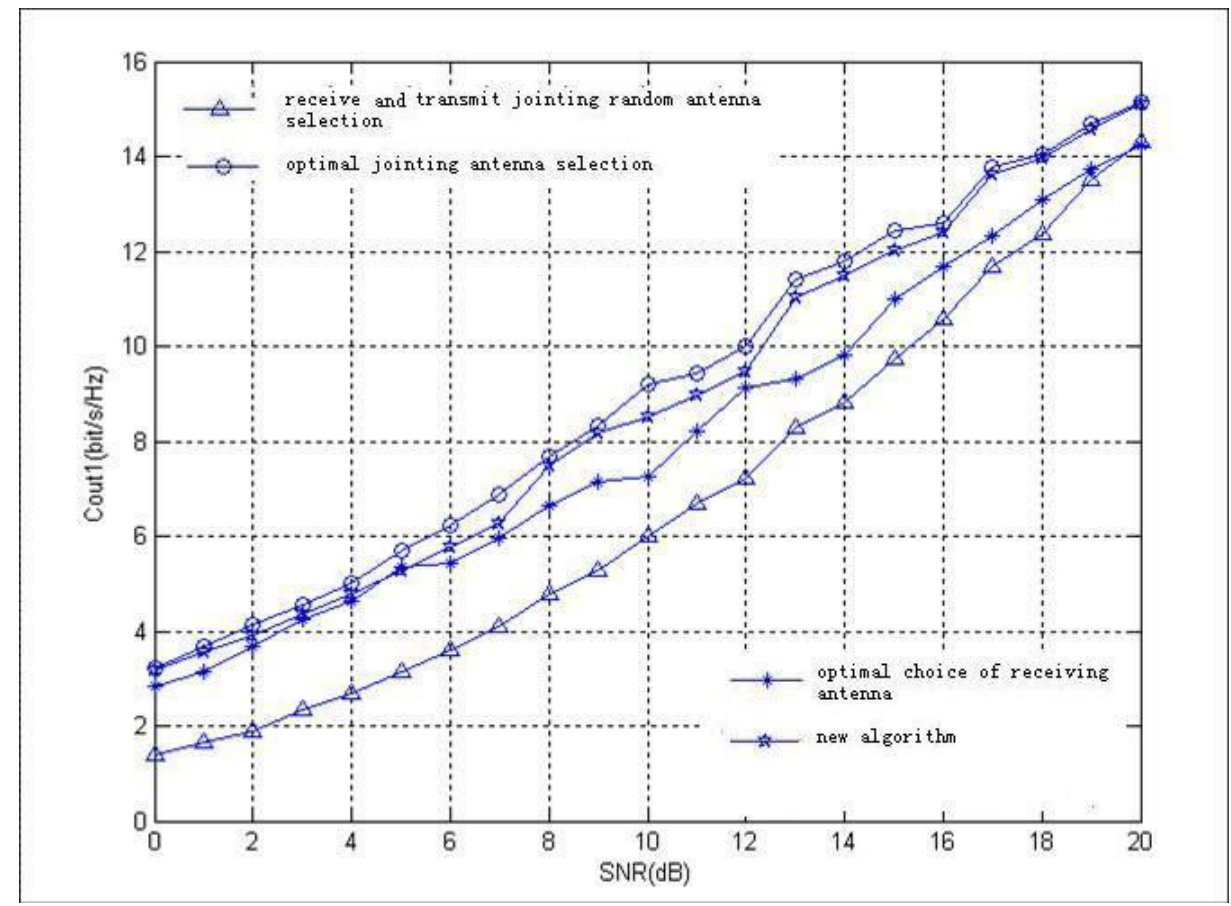

Fig. 3 . The $10 \%$ outage capacity when the correlation coefficient is 0.5

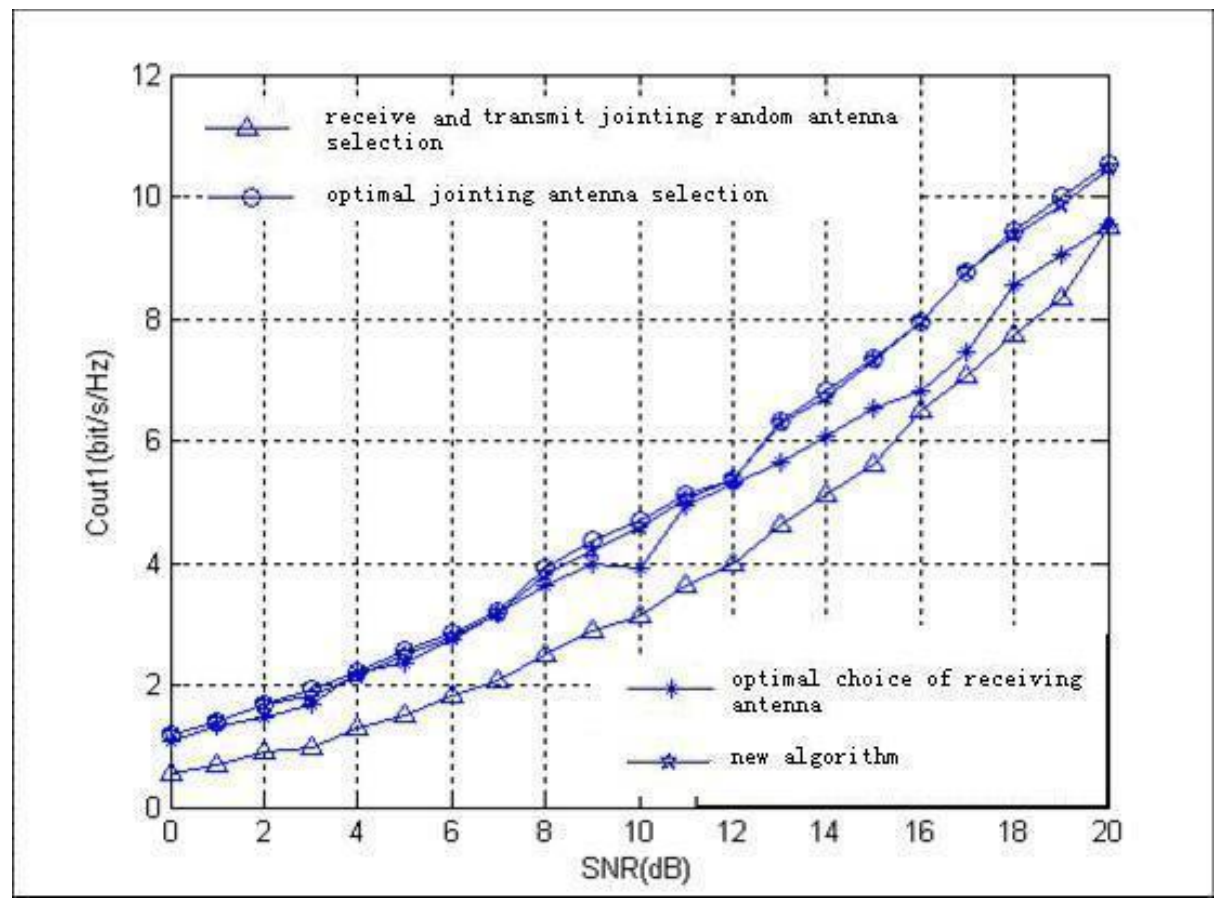

Fig. 4 . The $10 \%$ outage capacity when the correlation coefficient is 0.9 


\subsection{Analysis of the number of antenna}

From Fig 5 to 8 , it can be seen that the probability of the new algorithm to choose four antennas is higher when the correlated coefficient of the receiving antennas is smaller(Such as 0 or 0.2 ). On the contrary, the probability of the new algorithm to choose three or two antennas is improved greatly. After using the new algorithm for the analysis of system capacity when the correlation coefficient between antennas are different, we can get the following conclusions: the new algorithm achieves higher channel capacity by selecting more antennas and will be able to meet the requirements of the higher channel capacity by choosing fewer antennas ,thus it can reduce the number of radio links and simplifies the difficult of the subsequent demodulation and decoding. The characteristics of the new algorithm to change the number of antennas adaptively can improve the capacity of MIMO system and simplify equipment of the MIMO system.

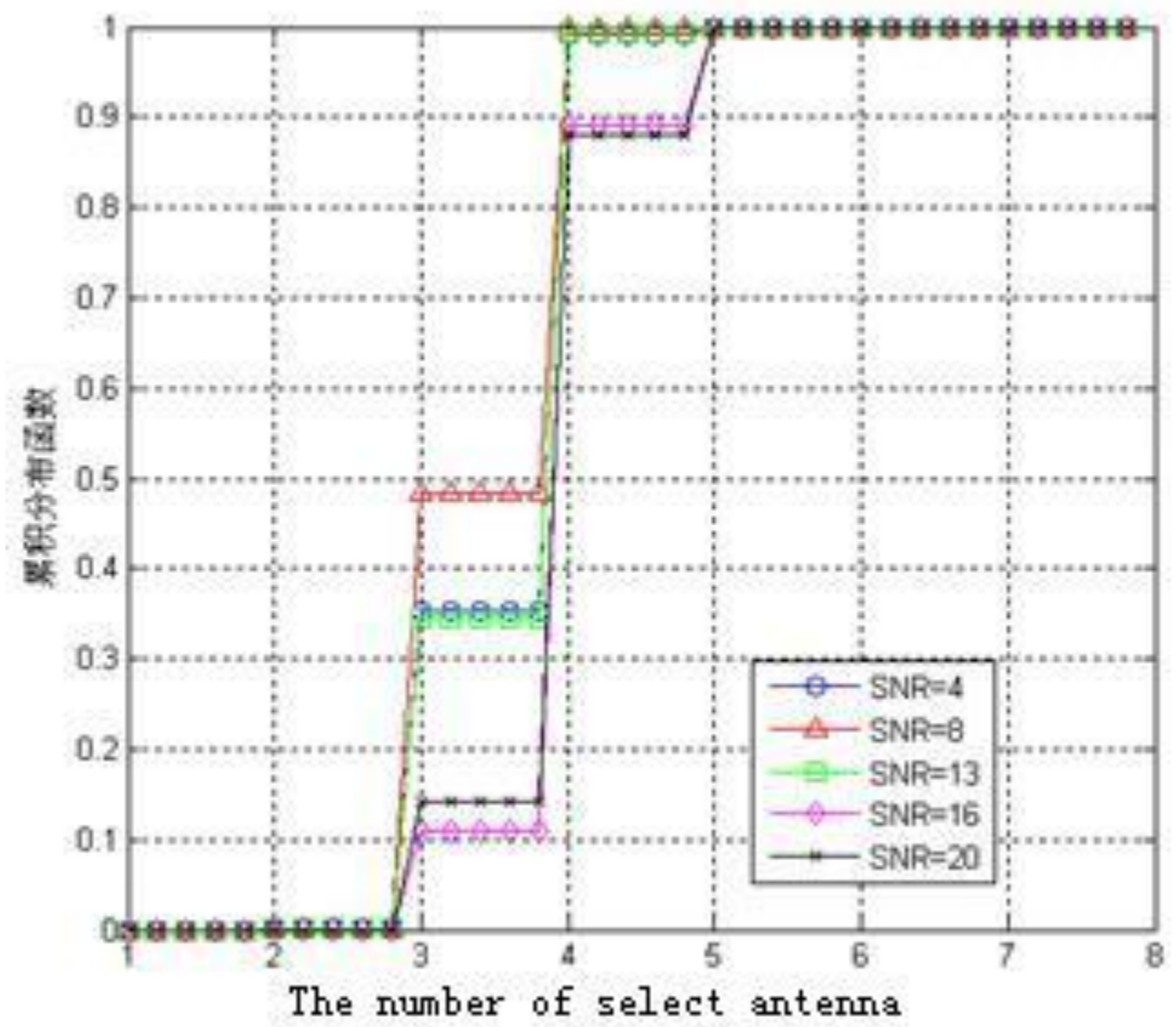

Fig. 5. The cumulative distribution of antenna number when the correlation coefficient is 0 


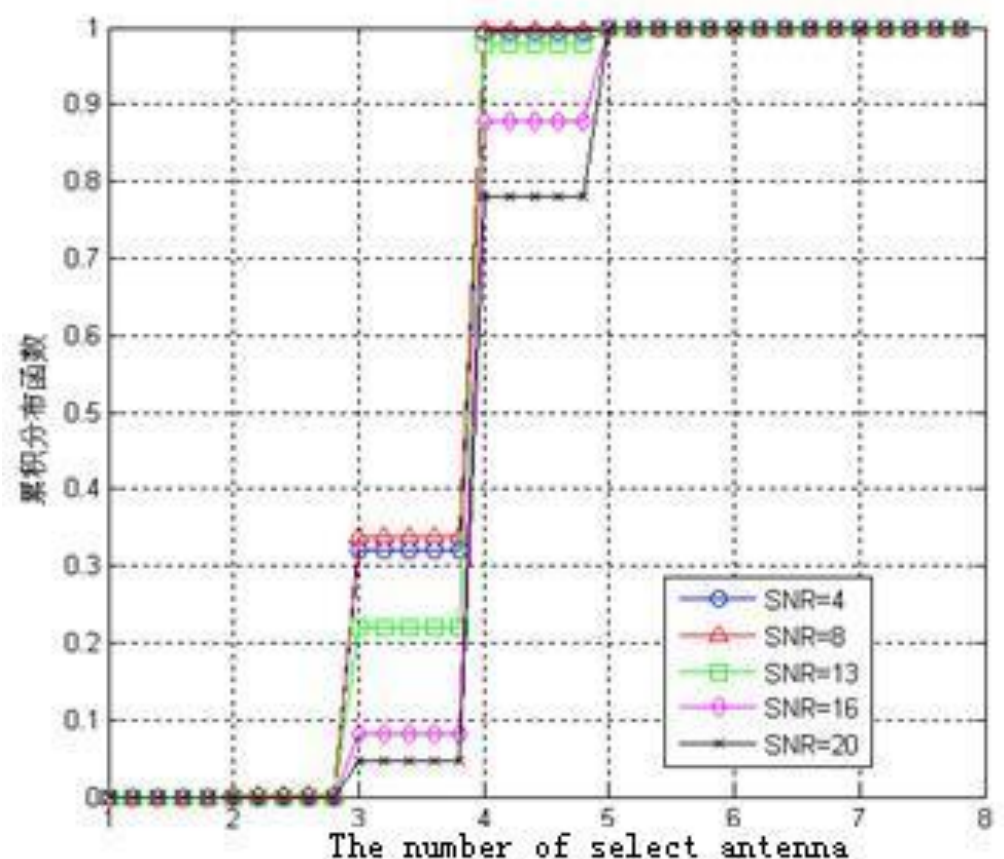

Fig. 6. The cumulative distribution of antenna number when the correlation coefficient is 0

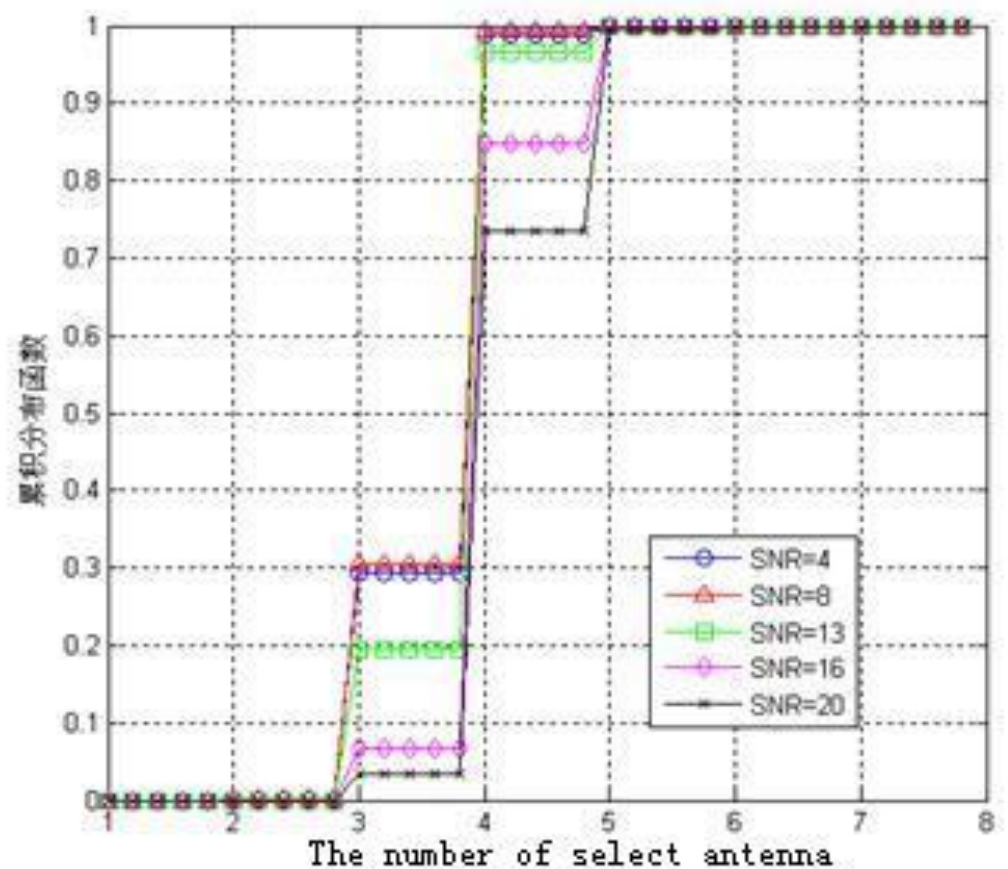

Fig. 7. The cumulative distribution of antenna number when the correlation coefficient is 0.5 


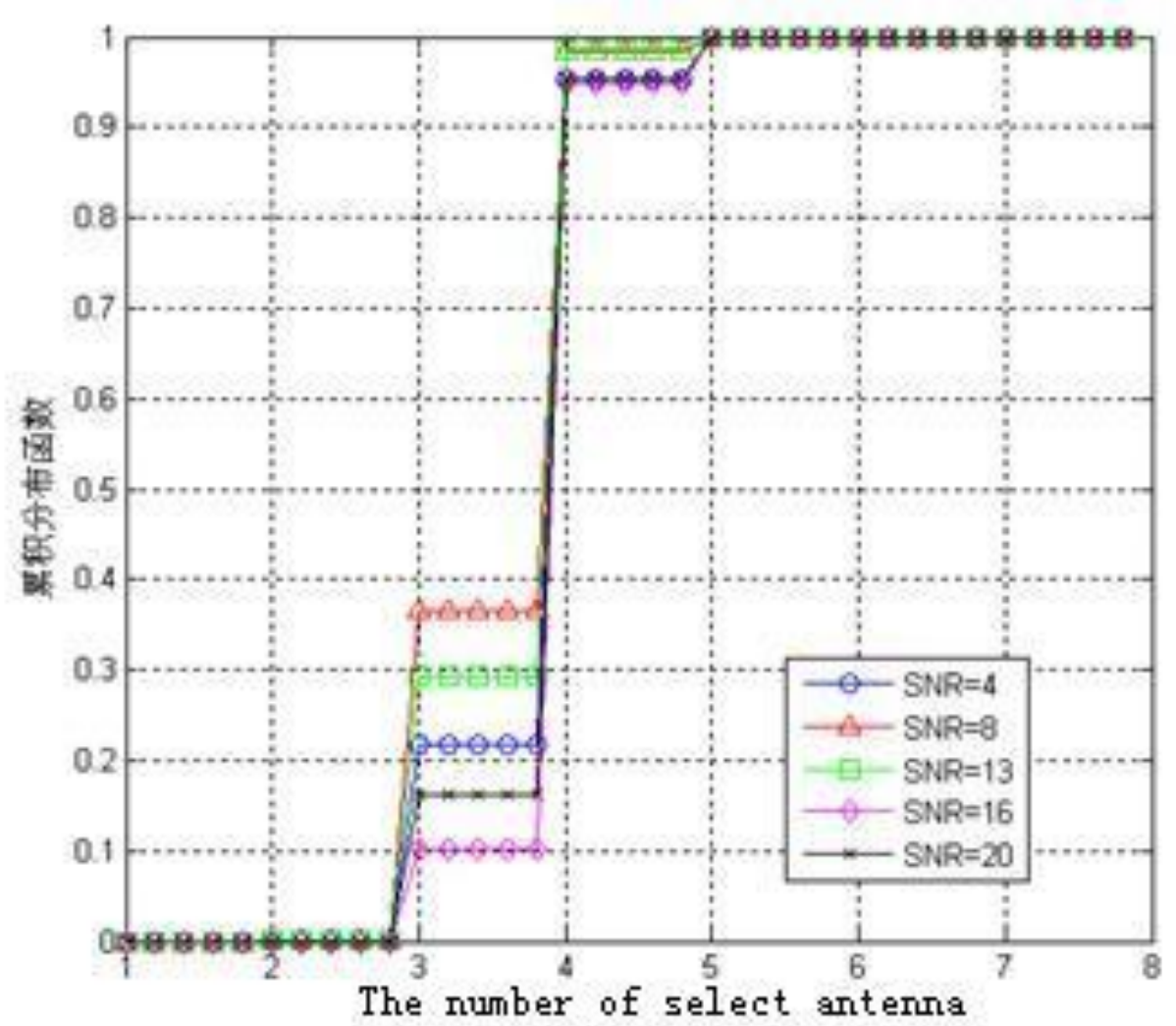

Fig. 8. The cumulative distribution of antenna number when the correlation coefficient is 0.9

\section{Conclusion}

In this paper, a new joint antenna selection algorithm was proposed which uses the idea of the maximum norm and the minimum correlation in the transmitter. It uses the Variable Antenna Selection which bases on capacity in the transmitter and use SNR to control the standards after choosing the channel capacity.

It is based on the different correlated coefficients in the receiver to select antennas. In general the algorithm is a variable number of joint antenna selection algorithm. The simulation results show that the new algorithm and the optimal joint antenna selection algorithm are similar in capacity regardless of correlated coefficient in the receiver. Compared with the receiver antenna selection it has a higher capacity when the number of antennas is constant. And the new algorithms can be adaptive to change the number of receiving antennas. The new algorithm achieves higher channel capacity by selecting more antennas and will be able to meet the requirements of the higher channel capacity by choosing fewer antennas, thus it can reduce the number of radio links and simplifies the subsequent demodulation and decoding. Therefore, the new algorithm can be applied to the wireless environments which have different relativity. Because of its strong self-adaptive linking, it reduces the number of the selected antenna and meets the system's channel capacity requirements. Therefore, it is more flexible in practical applications. In addition, the algorithm has low computational complexity, so it is able to meet the requirements of the real-time signal transmission. 


\section{Acknowledgements}

This work is sponsored by the National Basic Program of China (973 Program) (No.2007CB310605).

\section{References}

[1] Bluetest AB, Gothenburg, Sweden.Antenna performance characterization of multi-antenna terminals used in multiple-input-multiple-output communication, Antennas and Propagation Society International Symposium (APSURSI), IEEE 2010,11-17,1-4.

[2] Yi Liu, Yangyang Zhang,Chunlin Ji,Malik, W.Q. Edwards, D.J. Joint antenna slection for MIMO-OFDM system over spatially correlated channels. Wideband and Ultrawideband Systems and Technologies: Evaluating current Research and Development, IET Seminar on, 2008,6-6,3-5

[3] Molisch A F,Win M Z,Winters J H.Capacity of MIMO systems with antenna selection, IEEE International Conterence on Communications,2001,2,570-574.

[4] Gore D,Gorokhov A,Paulraj A.Joint MMSE verus V-BLAST and Antenna Selection,Signal Systems and Computers,2002,1(11),505-509.

[5] Gorokhov A,Antenna selection algorithms for MEA transmission systems, IEEE International Conference on Acoustics,Speech,and Signal Processing,2002,3,2857-2860.

[6] Gharavi-Alkhansari M,Gershman A B,Fast antenna subset selection in MIMO systems,IEEE Trans on Signal Processing,2004,52(2),339-347.

[7] Choi Y S,Molisch A F,Win M Z,etc,Fast algorithms for antenna selection in MIMO systems. IEEE 58th Vehicular Technology Conference, 2003, 3,1733-1737.

[8] Gu Zhaozhi, Liu Sheng. A modified denamic antenna selection algorithm for MIMO system, Signal Processing Systems (ICSPS), 2nd International Conference on2010.3, 294-297.

[9] L. Zheng, D. Tse, Diversity and multiplexing: A fundamental tradeoff in multiple-antenna channels, IEEE Trans on Inform Theory, 2003,49(5),1073-1096.

[10] [10] Joshi, Shreedhar A ,Rukmini, T S,Mahesh, H M ; System performance analysis of MIMO channel capacity with channel state approaches, Communication Control and Computing Technologies (ICCCCT), IEEE International Conference on 2010,7-9,71-74 